\title{
TRES COLORES
}

METODOLOGÍA COLABORATIVA

QUE PERMITA FACILITAR

LA VISUALIZACIÓN DE LOS

PROYECTOS SOCIALES Y DE PAZ,

REALIZADOS POR EL BARRISMO

\section{POPULAR}

THREE COLORS

COLLABORATIVE METHODOLOGY

THAT ALLOWS TO FACILITATE

THE VISUALIZATION OF THE

SOCIAL AND PEACE PROJECTS,

CARRIED OUT BY THE

HOOLIGANS

Diego Fernando Muñoz

\section{Muñoz}

Diseño Gráfico y

Multimedial, Universidad de Nariño, Pasto.

11 disgraficodm@gmail.com 


\section{Resumen}

El presente artículo aborda una experiencia de co-creación junto a la Banda Tricolor y la práctica del barrismo social en un barrio de Pasto, Nariño. El objetivo de acompañamiento desde el diseño es impulsar una nueva forma de barrismo que permite trabajar de la mano con las personas para lograr evidenciar el compromiso social que las diferentes barras populares del país efectúan en pro de la comunidad. Esto se hizo a partir del desarrollo de actividades de integración e inclusión barrial en contexto de pandemia a partir de la etnografía y el diseño co-creativo. El resultado de las actividades fueron regulares, ya que hubo una baja participación de la comunidad aledaña. Al

respecto se identifican las posibles causales y se analizan a detalle.
Abstract

This article addresses a co-creation experience with the Tricolor Band and the practice of social barrismo in a neighborhood of Pasto, Nariño. The objective of the accompaniment from the design is to promote a new form of neighborhood that allows working hand in hand with people to achieve evidence of the social commitment that the different popular bars of the country carry out in favor of the community. This was done from the development of neighborhood integration and inclusion activities in the context of a pandemic based on ethnography and co-creative design. The results of the activities were regular, as there was low participation from the surrounding community. In this regard, possible causes are identified and analyzed in detail. 

década de los noventa comienzan las primeras conformaciones de estos grupos de aficionados en Colombia, los cuales se agredían con enfrentamientos, mientras entonaban canciones de ofensa para los seguidores de otros clubes (Castro, 2016). Este tipo de prácticas generó un gran rechazo social a nivel nacional.

Los seres humanos en tanto seres sociales tienden a generar sociabilidades. Estas sociabilidades tienden a formar visiones de mundo, creencias y prácticas compartidas entre sus miembros. De ahí el imperativo social de que todo individuo pertenece a un grupo o varios grupos de individuos con quienes comparte dichas creencias y prácticas. Estas sociabilidades pueden ser partidos políticos, sectas religiosas o hinchadas de fútbol, por ejemplo (Clavijo, 2010). Lo importante de todas ellas, vale la pena reiterar es que todas tienen en común la construcción de ciertas visiones acerca de su propia realidad ya que no se puede ignorar la influencia de la acción colectiva en la elaboración de ideas dentro de una sociedad (Dávila y Londoño, 2003).

En cuanto a las barras bravas, entre sus principios ha estado la violencia como síntoma de la frustración social de los jóvenes que hacen parte de las mismas. Así, la proyección de frustraciones internas se ven representadas en la alteridad que constituye el límite de la identificación colectiva. Dicha problemática atraviesa un problema social de clase, ya que buena parte de estos jóvenes son de barrios populares y carecen de un futuro prometedor, así como vienen de un contexto de violencia familiar y local producto de una violencia estructural (Parada Dueñas, 2013). De cualquier modo, esto a conducido a la estigmatización social de estos jóvenes y su consiguiente rechazo generalizado por el resto de la sociedad.

Como respuesta a ello, en el caso colombiano y latinoamericano en general, ha venido surgiendo una nueva tendencia llamada barrismo social. Esta busca ser una alternativa a las dinámicas propias de las barras bravas. Desde la alta política, influenciada por las tendencias populares juveniles del barrismo social, se han formulado los objetivos de la política pública de Convivencia Ciudadana (Arroyo Chicaiza, 2014), los cuales se basan en la formulación e implementación de 
estrategias y capacidades públicas que ofrezcan alternativas de solución a las situaciones que hacen públicos los conflictos entre las personas pertenecientes a las barras de fútbol (Álvarez-Ossorio, 2013). Esta tiene como fin generar nuevos escenarios pedagógicos como respuesta a las conductas delictivas y de violencia producto de la violencia estructural y sistémica.De ahí surge la pregunta: ¿Cómo se puede dar a conocer las acciones de paz y los proyectos sociales en pro de la comunidad, que adelantan las barras populares? (Guerrero Sanzón, 2017).

El presente trabajo parte del estudio de caso de La Banda Tricolor de la ciudad de Pasto. De un tiempo para acá se viene hablando del barrismo social, el cual se ha visto manchado por la violencia que sigue presentándose en las barras populares del fútbol en Colombia. Sin embargo, en la actualidad y con la crisis social y económica producto de la pandemia del Covid-19, las barras de los clubes decidieron iniciar proyectos de labor social. Estos tienen como finalidad ayudar a familias de clases populares, como las de algunos integrantes, que padecen problemas financieros (El Espectador, 2020).

Este tipo de iniciativas que se han venido dando en varias ciudades de Colombia no son producto de la solidaridad espontánea del momento. Desde hace años, las múltiples hinchadas le han apostado al barrismo social, un proyecto que reúne a todos los integrantes de cada hinchada que busca realizar todo tipo de obras benéficas a favor de comunidades en condición de pobreza. Un ejemplo de este tipo de inicativas es el trabajo que se viene haciendo de la mano de la Escuela Musical Embajadora. Esta se encarga de guardar el instrumental de todas las barras de Millonarios y enseña a tocar instrumentos de percusión y viento a niños y jóvenes, da clases de arte para pintar banderas y murales, organiza entrega de regalos y eventos especiales en familia. En suma, la escuela viene promoviendo el arte, la cultura y el deporte para niños y jóvenes que no han tenido los recursos y la oportunidad de disfrutar de estas actividades. En otras palabras, este es el tipo de espacios que han creado las barras en Colombia para disminuir las problemáticas sociales y ser agentes activos en la transformación de la sociedad y promover una cultura de paz y convivencia (Osorio Guillott, 2020).

Ahora bien, es importante mencionar que a causa de la pandemia del Covid-19, el Centro de Investigación y Documentación para el Fútbol llevó a cabo el análisis trimestral de la labor adelantada por las barras, con el fin de abordar las acciones de solidaridad como respuesta colectiva que trasciende la rivalidad y resignifica la afición en beneficio del tejido social (Min. Deporte, 2020). Es así como el presente proyecto busca dar a conocer las actividades sociales que trabajan jóvenes integrantes de La Banda Tricolor en pro de la comunidad. Esta es una

primera prueba piloto de evaluación y ajuste de la metodología diseñada para facilitar la visualización de sus proyectos, los cuales manejan temáticas acerca de la violencia como un problema social, con el fin de cambiar la perspectiva que se tiene acerca del barrismo popular en la región. Para esto se hace necesario la creación de medidas educativas preventivas, para mejorar las acciones que inciden negativamente en el comportamiento de los integrantes de estos colectivos, y así poder establecer normas y otras alternativas frente a los mismos, acudiendo a estrategias de comunicación visual para lograr impactar en la comunidad y en la convivencia de la ciudad de Pasto.

De acuerdo a lo anterior se plantea la pregunta de investigación: ¿Cómo facilitar la visualización de las acciones de paz y los proyectos sociales que adelantan las barras populares? Esta se traduce en el objetivo general: Desarrollar una metodología abierta que permita facilitar la visualización de los proyectos sociales y de paz realizados por el barrismo popular. Este objetivo implica posteriormente validar con la comunidad la metodología propuesta aplicada a La Banda Tricolor y, luego, generar ajustes a la metodología, que permitan su implementación en otras barras populares.

\section{Método investigación}

En primera instancia, vale la pena alcarar que la presente investigación parte de un enfoque cualitativo, ya que se encuentra más preocupada por el entorno en donde suceden los hechos de la barra y centra su atención en aquellos sucesos en los cuales los integrantes de la barra se implican e interesan, evalúan y experimentan directamente. En este sentido, la observación es fundamental para lograr el objetivo y formular la metodología de la panera más apropiada de acuerdo a las prácticas y necesidades de La Banda Tricolor. Por ello, se escoge la etnografía como método de investigación. Esta se utiliza como una aplicación de metodología holística, la cual actúa en la formación constructivista, basada en la proposición de que toda persona encuentra su identidad, el significado y sentido de su vida a través de relaciones con la comunidad en determinados grupos particulares o en la sociedad en general. De 
acuerdo con lo anterior, se refirió el punto de análisis de la investigación a una de las tribus urbanas de la ciudad de Pasto, La Banda Tricolor, un grupo de barrismo popular.

De la mano de la etnografía, se plantea la metodología de diseño co-creativo propuesto por Paul Eaton (2011) que divide el proceso en tres etapas. En primer lugar, está la observación. En esta parte del proceso se incluye el otro, siendo la observación no solo un acto de contemplación fenomenológico sino un modo de interacción con el objeto de estudio; este proceso puede resultar ser el más complejo de todos. Se podría decir que en dicho ejercicio se incluye la labor de la etnografía. En cuanto a la segunda etapa, se trata de la generación. En ella, se lleva a cabo la construcción de la propuesta o un primer acercamiento a ella, tomando lo más relevante o necesario del proceso anterior. Por último, está lo que Eaton llama la construcción. Esta corresponde al análisis de cómo la obra encaja y es recibida por el medio en el cual se desenvuelve en la interacción con los usuarios.

La propuesta de Eaton, de acuerdo al contexto de la investigación, debe estar complementada por la proyección propuesta por Gui Bonsiepe (1978). Dicha propuesta metódica plantea no tres sino nueve pasos para el desarrollo del diseño. La primera etapa es la de identificar el problema: En esta etapa se realiza una búsqueda de información relevante que resulte útil en la toma de decisiones para solucionar alguna problemática. Segundo, está lo que Bonsiepe llama el análisis de necesidad: De acuerdo con los antecedentes recopilados, se lleva a cabo una descripción de la función y los objetivos generales del proyecto. Luego, se pasa a la definición del problema, en esta, se establecen los requerimientos específicos del proyecto, se especifica la problemática a intervenir y se hace una búsqueda de algunas soluciones. La quinta etapa comporta la síntesis de soluciones: En este punto se debe realizar una toma de decisiones en cuanto a la búsqueda de soluciones para la problemática escogida. En la etapa seis, desarrollo de diseño, se debe implementar algunas técnicas para la solución del problema, las visualizaciones de estas alternativas se deben evidenciar por medio de dibujos, esquemas, etc. En la fase de selección de alternativas se deben asignar valores a una lista de parámetros tales como complejidad, coherencia, etc. Posteriormente, en la etapa de comprobación de alternativas se lleva a cabo un testeo del diseño para verificar su viabilidad. Finalmente, se pasa al prototipo final que se trata de la reestructuración de la propuesta de solución a la problemática elegida, teniendo en cuenta la aceptación del público objetivo.

El papel del investigador será el de abordar el objeto de estudio con miras a comprender e interpretar una realidad que se está viviendo con las actividades de paz que se están trabajando para la comunidad. Esto incluye los proyectos sociales. Asimismo, este debe hacer un análisis e interpretación de la información proveniente del trabajo de campo, en el cual se deben utilizar datos (información verbal y no verbal) que consisten en experiencias textuales de los protagonistas del fenómeno y de la observación realizada en el ambiente natural para comprender lo que hacen, dicen y piensan sus actores.

La población seleccionada para el presente proyecto, son todos los integrantes de la barra popular La Banda Tricolor, de la ciudad de Pasto. Sin embargo, el trabajo principal se llevó a cabo junto con los integrantes del comité organizativo de La Banda Tricolor, que son entre 30 y 50 personas.

\section{Resultado}

Se desarrolla la metodología Tres colores que busca exponer a la comunidad la función social que adelanta La Banda Tricolor. Colectivo en el cual se materializa el resultado final de los diseños como aporte a la convivencia entre la comunidad y el barrismo popular en la ciudad de Pasto. Durante del desarrollo de la metodología, fue muy importante la aplicación de estrategias de comunicación visual. Estas últimas, surgen a partir de las actividades empleadas durante la ejecución del proyecto y ayudan a evidenciar el trabajo de este colectivo en la ciudad.

Figura 1 Infografía de Metodología Tres Colores

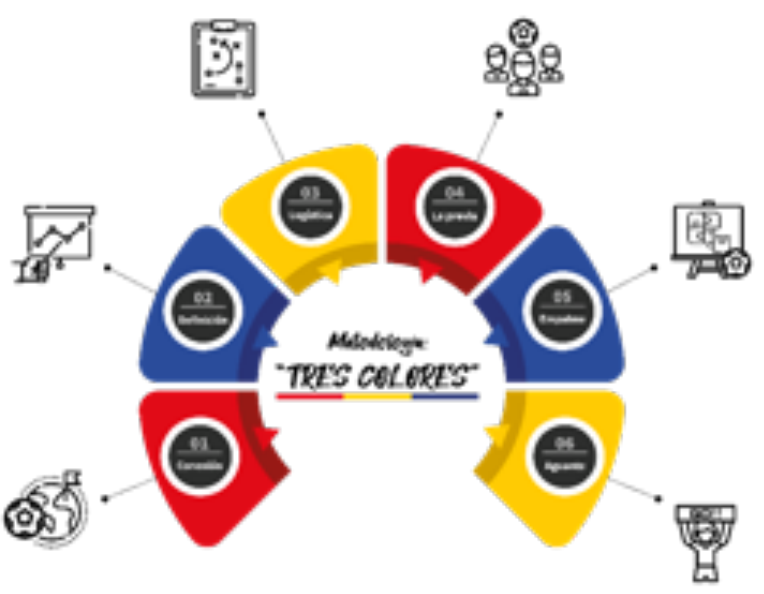


La metodología Tres colores se desarrolla como un trabajo colaborativo que sirve de facilitador en la construcción social. Esta contribuye a evidenciar la capacidad que tienen los integrantes de las barras populares para trabajar de manera colectiva en la resolución de problemáticas que afectan a la comunidad. En la actualidad el diseño está inmerso en todas partes, con una gran importancia a nivel estético y funcional. En efecto, la creación de dicho método es un ejemplo de ello. Este último debe ser entendido como un proceso de creatividad que se aplica al estudio de caso con el fin de hacer visible la función social que vienen desarrollando, lo que genera un gran impacto por medio de la innovación social. Su importancia dentro de su aplicación en el barrismo está encaminada en la búsqueda de soluciones para satisfacer necesidades reales de la comunidad co relación a alguna problemática específica, ya que todo gira en torno a la sociedad y precisamente para que también por medio del fútbol, las soluciones ayuden a mejorar su calidad de vida.

\section{¿Cómo funciona la Metodología?}

Su funcionamiento está relacionado con el trabajo colaborativo y el desing thinking, los cuales trabajan de manera complementaria, puesto que ambos siguen una serie de pasos en los cuales se exponen algunos aspectos importantes. A continuación, se describen los más importantes. El primero vendría a ser la generación de empatía que tiene como fin de entender las necesidades de las barras populares. El trabajo en equipo, etapa indispensable para el buen desarrollo de este método, ya que se valora la capacidad de los integrantes de las barras populares para aportar ideas de gran importancia en la búsqueda de la solución a la problemática fijada; cuanto más diverso sea el equipo de trabajo, mucho mejor, ya que esto permite reunir diferentes puntos de vista, conocimientos y experiencia. Para un completo empleo de la metodología es necesario la participación de una persona con conocimientos sobre la misma, para que pueda guiar el proceso. Por último, sigue la generación de prototipos. En ella se debe aprobar cualquier idea antes de su implementación, puesto que con este método se logra identificar fallas que se puedan solucionar en el proceso de desarrollo, antes de presentar la solución deseada. Durante esta etapa, hay que tener en cuenta los materiales que se vayan a utilizar como; hojas de papel, colores, marcadores, tijeras, cámara fotográfica, pegamento, además es importante que el espacio donde se vaya a desarrollar el prototipo sea adecuado para la realización de las actividades.

(

\section{Conclusiones}

Se identifican tres aspectos importantes a la hora de evidenciar la falta de participación de la comunidad con relación a los proyectos sociales y las actividades de paz llevadas a cabo por La Banda Tricolor en la ciudad de Pasto. En primer lugar, se identifica la mala organización interna, que hace que no haya interés de los integrantes de estos colectivos en las diferentes actividades sociales que se desarrollan; segundo, la falta de estrategias publicitarias que dificultan la propagación de la información hacia la comunidad; por último, el mal comportamiento de algunos de sus integrantes, el cual genera temor dentro y fuera de los escenarios deportivos. Si bien el fútbol es una pasión que mueve masas, el comportamiento inadecuado de algunos hace que se empañe la intensión de cambio y reivindicación de estos colectivos por medio de los proyectos sociales.

Sea como fuere, se puede decir que la creación e implementación de una metodología que contenga estrategias de comunicación incide positivamente, porque, en este caso particular, se logró difundir con mayor facilidad los proyectos sociales y las actividades de paz que realiza La Banda Tricolor y el barrismo popular en la ciudad de Pasto. Asimismo, se determinó que la implementación de la metodología, influyó en la difusión de los proyectos sociales y las actividades de paz que lleva a cabo La Banda Tricolor y su barrismo popular en la ciudad de Pasto, porque el diseño de identidad visual, Storytelling realizados, obtuvo una visión detallada y explicativa de los requisitos definidos, especificando su funcionamiento de acuerdo al estudio realizado sobre la percepción de la comunidad.

La construcción del diseño de la metodología de acuerdo a las necesidades de la comunidad objeto de estudio, se definió de acuerdo al método de investigación y el seguimiento de la metodología proyectual realizada en la etapa de diseño metodológico. Factor que permitió generar un impacto importante en la comunidad. 


\section{Referencias bibliográficas}

Arroyo Chicaiza, C. V. (2014). Barrismo social y colectivo barrista colombiano: Los antecedentes del diseño de una política pública (Tesis de pregrado). Universidad del Valle, Cali.

Álvarez-Ossorio, S. F. (1 de Diciembre de 2013). Fútbol y manipulación social. Análisis desde la sociología, la economía, medios de comunicación y la política. [Blog]. Recuperado de: https:// ssociologos.com/2013/12/01/futbol-y-manipulacion-socialanalisis-desde-la-sociologia-la-economia-medios-decomunicacion-y-la-politica/

Bonsiepe, G. (1978). Teoría y práctica del Diseño Industrial. Barcelona: Gustavo Gili.

Castaño Pérez, G. A., \& Uribe Aramburo, N. I. (2014). Barras bravas en el fútbol consumo de drogas y violencia. Medellín: Fundación Universitaria Luis Amigó.

Castro, J. A. (Agosto de 2016). La violencia del fútbol en Colombia. Palabras al margen. Recuperado de: http://palabrasalmargen. com/edicion-87/la-violencia-de [7] (2015). Identidad y rivalidad en una barra brava de Bogotá. Revista de Antropología y Sociología: Virajes, 17(1), 207-228.

Clavijo, J. (2010). Cantar bajo la anaconda: Un análisis sociocultural del barrismo en el fútbol. Bogotá, D.C: Pontificia Universidad Javeriana.

Dávila, A., \& Londoño, C. (2003). La nación baja un informe, fútbol e identidad nacional en Colombia, 1985 - 2000. En P. Alabarces, Futbologías: Fútbol, identidad y violencia en América Latina. Buenos Aires: CLACSO.

Dunning, E., Murphy, P. \& Williams, J. (1996). La violencia en los espectadores de fútbol. Hacia una explicación sociológica. En N. Elías, \& E. Dunning, Deporte y ocio en el proceso de la civilización (pp. 295-322). México D. F: Fondo de Cultura Económica.

Eaton, P. (2011). Co-creación y transdisciplina en procesos de Diseño. Recuperado de: https://wiki.ead.pucv.cl/Paul_Eaton:_Cocreaci\%C3\%B3n_y_transdisciplina_en_procesos_de_ dise\%C3\%B1o.

Fútbol HYE. (1 de Marzo de 2016). Fútbol: Su origen y evolución. [Blog]. Recuperado de https://futbolhye.wordpress.com
Gurrero Sanzón; G. (2017). Origen de La Banda Tricolor. (D. Muñoz, Entrevistador)

El Espectador. (Abril de 2020). Barras del fútbol colombiano donan mercados durante la cuarentena El Espectador. Recuperado de: https://www.elespectador.com/deportes/futbol-colombiano/ barras-del-futbol-colombiano-donan-mercados-durante-lacuarentena-articulo-914924/

Ministerio de Deporte. (2020). Barrismo social, eje del segundo boletín del Centro de Investigación y Documentación para el Fútbol de Mindeporte. (Boletín 2). Recuperado de: https://www.mindeporte. gov.co/sala_prensa/noticias_coldeportes/recursos_user/2020/IVC/ Septiembre/Boletin_Observatorio_Violencia_02.pdf

Osorio Guillott; A. (Mayo de 2020). El canto solidario de las barras en Colombia. El Espectador. Recuperado de: https://www. elespectador.com/deportes/futbol-colombiano/barrismosocial-en-tiempos-del-coronavirus/

Parada Dueñas, F. (2013). Barras bravas: Tensiones y convergencias desde una pesrpectiva híbrida. Revista de Ciencias Sociales, (31), 61-85.

Revilla, A. (2004). Rituales de agresión en subculturas juveniles y urbanas: Hooligans, Hinchas y Ultras. Cuadernos de realidades sociales, (45-46), 51-76.

Tribus Urbanas. (Agosto de 2015). Hooligans y ultras / Cultura hooligan. [Blog]. Recuperado de: https://todas-las-tribus urbanas.blogspot.com/2015/08/hooligans-y-ultras.html 

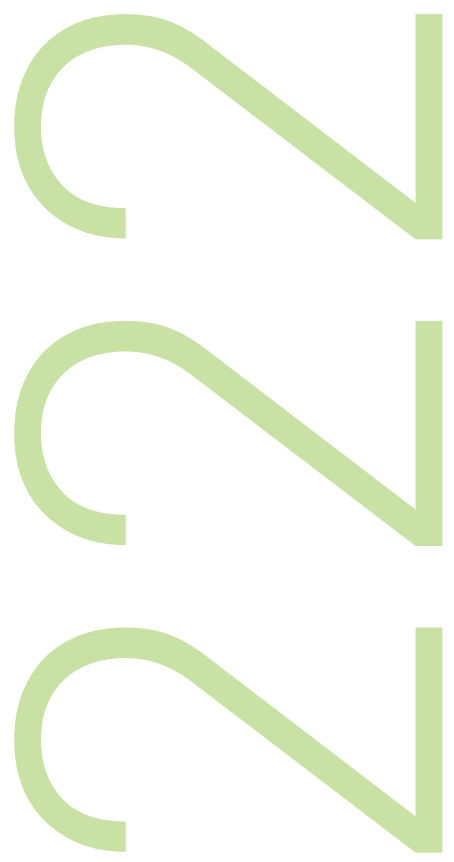